\title{
Humans' responses to novel stimulus compounds and the effects of training
}

\author{
EDMUND FANTINO and HERNÁN I. SAVASTANO \\ University of California, San Diego, La Jolla, California
}

\begin{abstract}
Twenty-four college students participated in an experiment with stimulus compounds. Subjects learned to discriminate color stimuli that correlated with varying probabilities of reinforcement. Reinforcement consisted of points. For all subjects, two colors signaled a .80 reinforcement probability, and two others signaled a .20 probability. For compound-trained subjects, a fifth compound stimulus (composed of a high-probability color and a low-probability color) was correlated with a .10 reinforcement probability. During testing, interspersed probe trials required subjects to choose between two alternatives: a compound stimulus and either one of its constituent stimuli. Compound-untrained subjects preferred the compound over either individual stimulus, thus showing response summation. However, compound-trained subjects, having had experience with an exemplar compound, showed significantly lower choice proportions for a test compound, indicating that subjects' responding to novel stimulus compounds is modifiable by experience with a single similar compound.
\end{abstract}

Our environment contains multiple sources of reinforcement that continuously control our behavior. Stimuli paired with reinforcing events signal their availability. Experiments with stimulus compounds study how these signals jointly affect behavior. Specifically, we assess the effect on responding of two simultaneously presented discriminative stimuli, the stimulus compound. The present experiment studied humans' responses to stimulus compounds and whether they could be altered by experience with similar compounds.

A typical compounding experiment initially fosters a discrimination between two previously neutral stimuli, each presented independently and associated with different rates of reinforcement. During testing, the discriminative stimuli are combined for the first time and in extinction. The amount of responding to the compound, measured by rate, magnitude, or probability of a response, is compared with the levels of responding controlled by the individual stimuli. Three outcomes are possible. In response summation, responding is greatest in the presence of the compound. The presence of both discriminative stimuli increases responding above the levels evoked by either stimulus alone. Response averaging occurs when responding to the compound is intermediate between the response levels evoked by the individual stimuli. Finally, response subtraction would occur when responding is lowest to the compound relative to either individual stimulus.

This research was funded by NIMH Grant MH-20752 and NSF Grant BNSn 91-08719 to the University of California, San Diego. We are grateful to Ben Williams for detailed criticisms throughout, J. R. Jones for helpful comments on an earlier draft of the manuscript, Will Wright for invaluable technical assistance, and Laura Hoffee for helping to conduct the experiment. Correspondence should be addressed to either author at the Department of Psychology, 0109, University of California, San Diego, La Jolla, CA 92093-0109 (e-mail: hsavasta@ucsd.edu).
Numerous studies on stimulus compounding support early assertions by Hull (1929) and Skinner (1957) that independent sources of response strength combine in an additive manner (see Weiss, 1972, for a thorough review). Original experiments employed the classical conditioning paradigm. Pavlov found summation of dogs' salivation to the simultaneous presentation of an odor and a mild shock (Kimble, 1961). With human subjects, Grings and O'Donnell (1956) and Hull (1940) showed significantly greater galvanic skin responses to a pair of conditioned stimuli than to each one separately. The finding apparently extends to operant procedures using nonhuman subjects (Miller, 1971; Miller \& Ackley, 1970; Wolf, 1963). For example, Weiss $(1967,1971)$ trained rats to respond to variableinterval food reinforcement schedules with a tone or light discriminative stimulus. During testing, all subjects showed summated response rates to the simultaneous presentation of tone and light. There have been no studies of operant stimulus compounding using human subjects. This is unfortunate since cognitive "averaging" models proposed in the area of judgment and decision making (Anderson, 1981) suggest that humans should not summate stimulus values.

The explanation of evidence for operant response summation has relied on the assumption that increases in response rate reflect changes in response strength. However, molecular contingencies that affect response rate may not affect value (Nevin, 1988). Because choice measures often show greater sensitivity to reinforcement manipulations, preference more accurately represents response strength and value (Herrnstein, 1970). With stimulus compounds, if stimulus values combine in an additive manner, then subjects should prefer a compound over either of its constituent stimuli. The present experiment used a choice procedure to assess whether response summation occurs with human subjects in an instrumental task. 
We trained human subjects in a delayed-matching-tosample (DMTS) task. They responded (on a keyboard) to stimuli associated with varying conditional probabilities of reinforcement. Stimuli consisted of color images displayed on a computer monitor, and reinforcement consisted of points. During testing, interspersed probe trial choices were presented, during which choice responses produced no immediate reinforcement. Subjects chose between individual stimuli and compounds of two stimuli. Preference for the compound with respect to its constituents was assessed. The three possible outcomes corresponded to different choice patterns. Summation occurred when subjects chose the compound over both the high- and the lowprobability constituents. Averaging occurred when subjects chose the compound over the low-probability constituent, but also chose the high-probability constituent over the compound. Response subtraction occurred if subjects preferred both of the individual constituents over their compound.

A second objective was to determine whether experience with similar compounds alters responding to future compounds. All subjects received exposure to four color stimuli. However, half of the subjects also received exposure to a compound stimulus consisting of two of those colors during the training phase. This compound produced the lowest conditional reinforcement probability of all the training stimuli. During probe trials, a different compound was tested. Decreased preference for the test compound would suggest that responding to compounds is controlled by prior experience.

\section{METHOD}

\section{Subjects}

Twenty-four undergraduates ( 17 female, 7 male) participated as subjects through three 40-min sessions in exchange for course credit and either $\$ 5$ or $\$ 10$, which they received after completing the experiment.

\section{Apparatus}

Experimental sessions took place in a small room, which contained a desk, a chair, and an OEI 386/33 compatible personal computer to control experimental events. Stimuli were displayed on a $14-\mathrm{in}$. $(35.6-\mathrm{cm})$, high-resolution color monitor (IMPRESSION, $.39 \mathrm{dp}$ ). Colors were selected from the standard 16-color VGA palette.

\section{Procedure}

Initially, the subjects were trained in a DMTS task. Each trial began with a sample stimulus, centered on the screen. The sample remained on the screen until the subject responded once by pressing the letter $G$ on the keyboard. Following a $1-\mathrm{sec}$ interstimulus interval (ISI), when the screen was dark, two choice stimuli appeared on the left and right halves of the screen, horizontally aligned and $7 \mathrm{~cm}$ apart from each other. The subjects pressed the letter $F$ to choose the stimulus on the left half of the screen or $\mathrm{H}$ for the stimulus on the right. (The letters correspond to left and right spatial locations on the keyboard.) One choice stimulus always matched the sample and was the correct alternative. Its position alternated pseudorandomly over trials in order to prevent side bias. Responses that matched the sample produced a reinforcer with varying probability $(.80, .20$, or .10$)$, depending on the stimulus. Reinforcement consisted of an immediately contingent message typed on the screen which stated, "Congratulations! You have just earned a point. Your point total thus far is $X$ ". The number $(X)$ incremented by one each time a point was earned. The reinforcer lasted $1 \mathrm{sec}$ and preceded a $1-\mathrm{sec}$ intertrial interval (ITI). A correct choice that did not produce a reinforcer led to a 2-sec ITI, during which the screen was dark. The other choice stimulus, selected pseudorandomly, could be any other stimulus from the set. Choosing the incorrect alternative never produced a reinforcer and preceded a 2-sec ITI before the start of the next trial.

Training. For all subjects, the first session entailed training on the DMTS task with four different stimuli: red, yellow, green, and blue. Each stimulus in the set was equally likely $(p=.25)$ to appear as the sample. Stimuli had the shape of a half-circle (radius $=1.75 \mathrm{~cm}$ ), which faced up or down in pseudorandom fashion. Two colors, $C_{1}$ and $C_{2}$, produced reinforcement with a .80 probability when the subjects matched to sample. Matching the other two colors, $\mathrm{C}_{3}$ and $\mathrm{C}_{4}$, produced a reinforcer with a .20 probability. Thus, two stimuli in the set correlated with a relatively high conditional probability of reinforcement, and the remaining two stimuli predicted a relatively low conditional probability. Stimulus colors were counterbalanced across subjects. The first session ended after 360 trials, which the subjects usually completed within $35 \mathrm{~min}$

Prior to their second session, the subjects were randomly assigned to either the compound-untrained or compound-trained group. Compounduntrained subjects' second session was identical to their first. Compoundtrained subjects, however, were presented a fifth stimulus during their second training session, making the probability of occurrence of each sample .20. The added stimulus consisted of a compound of two colors, $\mathrm{C}_{2}$ and $\mathrm{C}_{4}$ (a high-probability color and a low-probability color). The two half-circles combined to form a full circle, half of one color and half the other. This compound stimulus delivered a reinforcer with the lowest of all reinforcement probabilities, .10 , when matched to sample. All second sessions lasted 440 trials, usually about $40 \mathrm{~min}$.

Probe trials. During the third test session, we pseudorandomly interspersed probe trials. Otherwise, this session was similar to the second session for each group. In a probe trial, no sample stimuli occurred. The trial simply began with the presentation of two choice stimuli. The subjects chose between the alternatives by pressing $\mathrm{F}$ or $\mathrm{H}$, as in training trials. Because the dependent measure was preference during probe trials, choices did not produce reinforcers. Neither the reinforcement message nor the point counter appeared during the test session. A 2-sec darkscreen ITI followed each choice response.

Five types of probe trials were presented: $C_{1}$ versus $C_{3}$, pitting a highprobability stimulus against a low-probability stimulus, to ensure training had exerted adequate discriminative control; $C_{1} / C_{3}$ versus $C_{3}$, a high/low compound against a low-probability stimulus; $C_{1} / C_{3}$ versus $C_{1}$, the compound against a high-probability stimulus; $C_{1} / C_{3}$ versus $C_{1} /$ new, the compound against a compound of a high-probability color and a novel, gray color; and $C_{3}$ versus new (gray), a low-probability stimulus versus the novel stimulus. The last two probe types are controls (see Discussion section below). Testing entailed 30 presentations of each probe type, adding 150 trials to the last session. Probe trials used only red, green, and gray colors. None of the subjects had any experience with the $\mathrm{red} / \mathrm{green}$ compound or the gray stimulus prior to testing.

Instructions. All subjects were given standard instructions. For the first two sessions, the subjects were instructed to try to earn points. They were told which keys on the keyboard were active, and they were also told that color, not shape or orientation, was the relevant stimulus dimension. Instructions never addressed the probabilities of reinforcement associated with each stimulus. Prior to the test session, subjects were instructed as follows:

Your task today is similar to the first two sessions. However, there are some differences. Along with the types of trials you've seen previously, you will also see some new trials in which there is no first image [the sample]. You will simply see two color images. Always choose the one that you think is most likely to earn you a point.

The subjects were also told that points could be earned during probe trials, but that they would be tallied at the end of the session and that neither the reinforcer message nor the point counter would appear during the session. Also, the subjects were told that they could earn either a $\$ 10$ reward for beating the current high score or a $\$ 5$ reward otherwise. Following experimental sessions, completion of a brief questionnaire and debriefing about the experiment ended subject participation.

\section{RESULTS}

The programmed reinforcement probabilities closely matched those obtained as all subjects learned to match to 
sample. The mean percent correct was $95 \%$. Averaged over subjects and sessions, the obtained reinforcement probabilities were .79 for high-probability stimuli, .20 for low-probability stimuli, and .08 for the trained compound (compound-trained subjects only).

Table 1 presents individual-subject choice proportions during probe tests, along with group means and standard errors. Choice proportions represent the number of trials (out of 30) in which the stimulus noted first in each column heading was chosen over the other alternative in that probe type. Choice proportions in $C_{1}$ versus $C_{3}$ (high vs. low) probes indicated a sufficient discrimination between high-probability and low-probability stimuli. Mean choice proportion for compound-untrained subjects was .75 for the high-probability stimulus. Compound-trained subjects responded similarly, choosing $C_{1}$ over $C_{3}$ on $79 \%$ of trials. Data analyzed do not include those of 4 subjects who failed a minimum discrimination criterion of .50.

\section{Compound-Untrained}

Compound-untrained subjects preferred the compound over low-probability stimuli $\left(\mathrm{C}_{1} / \mathrm{C}_{3}\right.$ vs. $\left.\mathrm{C}_{3}\right)$ with a mean choice proportion of 88 . Mean preference for the compound over high-probability stimuli $\left(\mathrm{C}_{1} / \mathrm{C}_{3}\right.$ vs. $\left.\mathrm{C}_{1}\right)$ was .69 . In $\mathrm{C}_{1} / \mathrm{C}_{3}$ versus $\mathrm{C}_{1} /$ new probes, subjects preferred a high/low compound over a high/new compound (.73). The subjects also chose low-probability stimuli over the novel stimulus $\left(C_{3}\right.$ vs. new) on $82 \%$ of trials. The pattern of choices exemplified by the group means implies response summation,

Table 1

Individual and Group Mesn Choice Proportions for the First Stimulus Denoted in the Column Heading of Each Probe Type

\begin{tabular}{|c|c|c|c|c|c|}
\hline \multirow[b]{2}{*}{$\underline{\text { Subject }}$} & \multicolumn{5}{|c|}{ Probe Type } \\
\hline & $\mathrm{C}_{1}$ vs. $\mathrm{C}_{3}$ & $\begin{array}{l}\mathrm{C}_{1} / \mathrm{C}_{3} \\
\text { vs. } \mathrm{C}_{3}\end{array}$ & $\begin{array}{l}\mathrm{C}_{1} / \mathrm{C}_{3} \\
\text { vs. } \mathrm{C}_{1}\end{array}$ & $\begin{array}{c}\mathrm{C}_{1} / \mathrm{C}_{3} \\
\text { vs. } \mathrm{C}_{1} / \text { new } \\
\end{array}$ & $\mathrm{C}_{3}$ vs. new \\
\hline \multicolumn{6}{|c|}{ Compound-Untrained } \\
\hline S1 & 0.73 & 0.67 & 0.63 & 0.60 & 0.63 \\
\hline S2 & 0.97 & 0.97 & 0.03 & 0.67 & 0.87 \\
\hline S3 & 0.57 & 0.77 & 0.87 & 0.97 & 0.93 \\
\hline S4 & 0.90 & 0.83 & 0.10 & 0.07 & 1.00 \\
\hline S5 & 0.97 & 0.83 & 0.93 & 0.93 & 0.97 \\
\hline S6 & 0.63 & 0.87 & 0.83 & 0.73 & 0.60 \\
\hline S7 & 0.90 & 0.97 & 0.97 & 0.97 & 0.93 \\
\hline S8 & 0.60 & 0.97 & 0.80 & 0.57 & 0.47 \\
\hline S9 & 0.67 & 0.93 & 0.87 & 1.00 & 1.00 \\
\hline$\$ 10$ & 0.57 & 0.97 & 0.90 & 0.83 & 0.83 \\
\hline$M$ & 0.75 & 0.88 & 0.69 & 0.73 & 0.82 \\
\hline$S E M$ & 0.06 & 0.03 & 0.11 & 0.09 & 0.06 \\
\hline \multicolumn{6}{|c|}{ Compound-Trained } \\
\hline S11 & 0.60 & 0.03 & 0.10 & 0.93 & 0.97 \\
\hline $\mathrm{S} 12$ & 1.00 & 0.10 & 0.00 & 0.97 & 1.00 \\
\hline S13 & 1.00 & 0.17 & 0.13 & 1.00 & 1.00 \\
\hline S14 & 0.93 & 0.87 & 0.03 & 0.00 & 0.00 \\
\hline S15 & 0.57 & 0.63 & 0.57 & 0.57 & 0.40 \\
\hline S16 & 0.70 & 0.67 & 0.50 & 0.87 & 0.83 \\
\hline S17 & 0.97 & 0.90 & 0.00 & 0.03 & 0.07 \\
\hline S18 & 0.67 & 0.60 & 0.63 & 060 & 0.27 \\
\hline S19 & 077 & 0.07 & 0.00 & 0.90 & 0.97 \\
\hline $\mathrm{S} 20$ & 0.73 & 0.20 & 0.07 & 1.00 & 1.00 \\
\hline$M$ & 0.79 & 0.42 & 0.20 & 0.69 & 0.65 \\
\hline$S E M$ & 0.06 & 0.11 & 0.09 & 0.13 & 0.14 \\
\hline
\end{tabular}

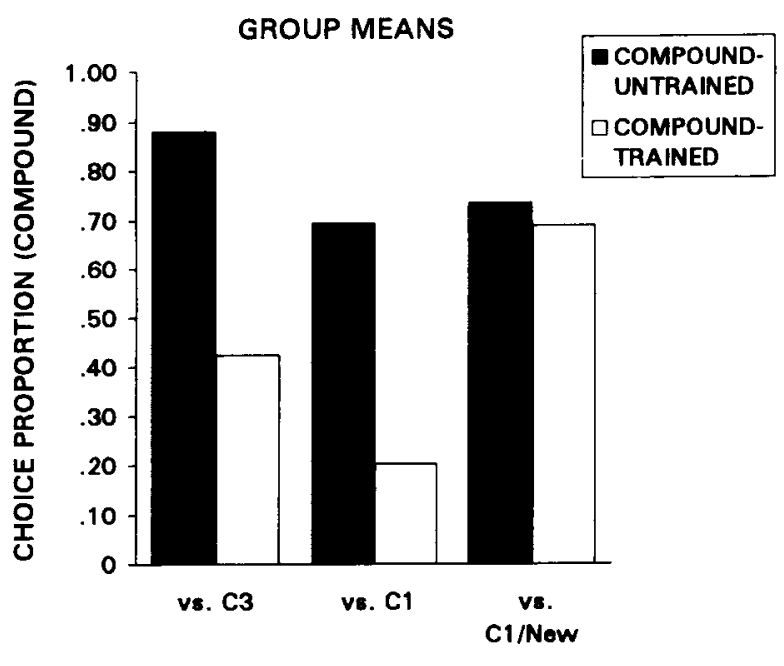

PROBE TYPE

Figure 1. Mean choice proportions during probe testing for each group of subjects. Preference is calculated for the compound, which consisted of one stimulus correlated with a high probability of reinforcement and one correlated with a low probability of reinforcement. The other alternative in each type of probe trial was: $C_{3}$, a low-probability stimulus; $C_{1}$, a high-probability stimulus; and $C_{1}$ /new (a high-probability stimulus and a novel stimulus).

preference for the compound over its constituents. Group results clearly reflected individual performance. Out of 10 subjects, 8 showed response summation.

\section{Compound-Trained}

Figure 1 displays mean choice proportions for both groups of subjects in three critical probe types: $\mathrm{C}_{1} / \mathrm{C}_{3}$ versus $C_{3}, C_{1} / C_{3}$ versus $C_{1}$, and $C_{1} / C_{3}$ versus $C_{1} /$ new. Choice proportions above .50 indicate preference for the compound $\left(\mathrm{C}_{1} / \mathrm{C}_{3}\right)$. Trained subjects' choice proportions differed from those of compound-untrained subjects in the first two probe types $[F(1,18)=23.66, p<.01]$.

Specifically, preference for the compound $\left(\mathrm{C}_{1} / \mathrm{C}_{3}\right)$ over the low-probability stimulus $\left(\mathrm{C}_{3}\right)$ dropped to .42 , significantly different from the compound-untrained group mean of $.88[F(1,18)=16.05, p<.01]$. Similarly, preference for $\mathrm{C}_{1} / \mathrm{C}_{3}$ over the high-probability stimulus $\left(\mathrm{C}_{1}\right)$ decreased to .20 from the compound-untrained subjects' $.69[F(1,18)=$ $13.05, p<.01]$. Individual-subject data also reflected the main effect, with 5 out of 10 subjects showing response subtraction, meaning that the individual stimuli were preferred over their compound. Of the other 5 subjects, only 2 (S15 and S18) showed choice patterns that resembled summation. However, both preferred the new stimulus over the lowprobability stimulus, suggesting that novelty controlled their choices.

\section{DISCUSSION}

Our results replicated the response summation previously found with nonhumans (and humans with Pavlovian procedures). Individually, 8 out of 10 compound-untrained subjects showed response summation. All 10 subjects chose the compound more frequently than its low-probability 
constituent, even though they had never seen the compound during training and, therefore, never earned a point from choosing it. Eight out of 10 subjects also preferred the compound over its high-probability constituent. Notice that preference for the compound depended on which alternative competed against it. As expected, means across subjects from both groups showed greater choice proportions for the compound when pitted against its low-probability constituent than when pitted against its high-probability constituent (Figure 1).

Performance in two control probe types $\left(C_{1} / C_{3}\right.$ vs. $C_{1} /$ new, $C_{3}$ vs. new) rejected some alternative interpretations of our results. Recall that compound-untrained subjects did not see the compound until probe trials began. If novelty controlled preference, the high/new compound $\left(C_{1} /\right.$ new $)$ should have been preferred since it was at least as novel as the high/low compound $\left(\mathrm{C}_{1} / \mathrm{C}_{3}\right)$. Instead, untrained subjects strongly preferred the latter. Similarly, the subjects should have chosen the novel stimulus over low-probability stimuli if novelty were a factor, but they did not. Second response summation might have resulted because the compound consisted of two stimuli rather than one, simultaneously changing overal stimulus intensity and number of constituent stimuli. If so, compounduntrained subjects should have been indifferent between the high/low compound and the high/new compound since both alternatives consisted of two constituents. The obtained results imply that preference for the compound went beyond the saliency of two-component stimuli.

The tendency of human subjects to evince response summation contrasts with many results in the area of human judgment and decision making. In several verbal tasks, subjects seem to employ averaging strategies to integrate stimulus values (Anderson, 1981). An attempt to reconcile the two findings would be difficult given the numerous methodological differences. In any event, our results with compoundtrained subjects suggest that the response summation pattern can be modified with training. Compound-trained subjects had received exposure to an exemplar (yellow/blue) compound correlated with infrequen payoff. Overall, compound-trained subjects tended to avoid choosing the test (red/green) compound when compared with compound-untrained subjects. As Table 1 shows, 5 out of 10 compound-trained subjects showed extreme preferences for the individual stimuli over their compound. Notice that none of the compound-untrained subjects showed similar response subtraction. Although training did not affect all subjects' choices, Figure 1 reflects a substantial (and statistically significant) effect of training. Apparently, the response to a novel compound is at least partially modifiable by previous experience with just one similar compound.

When interpreting data from human subjects, instructional control is often a concern. Although the instructions were explicit (see our Method section), perhaps the subjects believed their task during probe trials was to guess which sample had been omitted. If so, compound-untrained subjects might choose two-constituent stimuli to increase their chances of being correct, thus showing response summation. Similarly, because compounds occurred in only $20 \%$ of training trials, compound-trained subjects might prefer the simple stimuli in probe tests, thus showing response subtraction. However, the argument is weakened by the responses obtained to the postexperiment questionnaire and interview, which indicated that all subjects had understood the task correctly.

The relevance of our findings to research on negative patterning and configural conditioning merits discussion. Negative patterning refers to the relatively weak response evoked by a compound stimulus repeatedly presented in extinction (e.g., Kehoe \& Graham, 1988). The finding is held as evidence for configural conditioning since the response to the compound cannot be due simply to the summation of independent response strengths (or atomistic conditioning). Data from compound-trained subjects are consistent with the notion of configural conditioning. In part, these subjects may have learned to avoid the trained compound, a response that later generalized to the test compound, thus resulting in response subtraction. However, the subjects' preference for the high/low compound over a high/new compound $\left(C_{1} / C_{3}\right.$ vs. $C_{1} /$ new $)$ suggests some role of atomistic conditioning. Other studies confirm that both types of conditioning processes may operate in compounding experiments (Kehoe \& Graham, 1988; Kehoe, Horne, Horne, \& Macrae, 1994).

In summary, human subjects responded to compound stimuli more than to either of the component stimuli (summation); however, when given training with a single compound associated with a lower rate of reinforcement than either of the individual component stimuli, they showed significantly less responding to novel compounds than did subjects without this experience. This flexibility may be understood in terms of our need to adapt to changes in the environment. Perhaps summation is more frequently observed because, in everyday situations, the appearance of two signals for reinforcement often predicts an increased availability of reinforcement. However, because summation is not always an optimal strategy, the way we respond to compound stimuli must be susceptible to changing contingencies.

\section{REFERENCES}

ANDERSON, N. H. (1981). Foundations of information integration theory. New York: Academic Press.

GRINGS, W. W., \& O'DoNNELL, D. E. (1956). Magnitude of response to compounds of discriminated stimuli. Journal of Experimental Psychology, 52, 354-359.

HERRNSTEIN, R. J. (1970). On the law of effect. Journal of the Experimental Analysis of Behavior, 13, 243-266.

HuLL, C. L. (1929). A functional interpretation of the conditioned reflex. Psychological Review, 36, 498-511.

HuLL, C. L. (1940). Exploration in the patterning of stimuli conditioned to the GSR. Journal of Experimental Psychology, 27, 95-110.

KehoE, E. J., \& GRAhaM, P. (1988). Summation and configuration: Stimulus compounding and negative patterning in the rabbit. Journal of Experimental Psychology: Animal Behavior Processes, 14, 320-333.

Kehoe, E. J., Horne, A. J., Horne, P. S., \& Macrae, M. (1994). Summation and configuration between and within sensory modalities in classical conditioning of the rabbit. Animal Learning \& Behavior, 22, 19-26.

KIMBLE, G. H. (1961), Hilgard and Marquis' conditioning and learning New York: Appleton-Century-Crofts.

MiLler, L. (1971). Compounding of discriminative stimuli from the same and different modalities. Journal of the Experimental Analysis of Behavior, 16, 337-342.

MILLER, L., \& ACKLEY, R. (1970). Summation of responding maintained by fixed-interval schedules. Journal of the Experimental Analysis of Behavior, 13, 199-203.

Nevin, J. A. (1988). Behavioral momentum and the partial reinforcement effect. Psychological Bulletin, 103, 44-56

SkinNeR, B. F. (1957). Verbal behavior. New York: Appleton-CenturyCrofts.

WEIss, S. J. (1967). Free-operant compounding of variable-interval and low-rate discrimination stimuli. Journal of the Experimental Analysis of Behavior, 10, 535-540.

WEISs, S. J. (1971). Discrimination training and stimulus compounding: Consideration of non-reinforcement and response differentiation consequences of extinction. Journal of the Experimental Analvsis of Behavior, 15, 387-402.

WEISs, S. J. (1972). Stimulus compounding in free-operant and classical conditioning: A review and analysis. Psychological Bulletin, 78, 189-208.

WoLF, M. M. (1963). Some effects of combined S 's. Journal of the Experimental Analysis of Behavior, 6, 343-347.

(Manuscript received August 8, 1994; revision accepted for publication April 10, 1995.) 